\title{
Central meson production in diffractive reactions
}

\author{
Antoni Szczurek ${ }^{* \dagger}$ \\ Institute of Nuclear Physics PAN, ul. Radzikowskiego 152 \\ PL-31-342 Cracow, Poland \\ and \\ University of Rzeszów, ul. Rejtana 16 \\ PL-35-959 Rzeszów, Poland \\ E-mail: antoni.szczurek@ifj.edu.pl
}

I discuss double-diffractive (double-elastic) production of the $\eta^{\prime}$ and $\eta_{c}$ mesons in the $p p \rightarrow$ $p X p$ reaction within the formalism of unintegrated gluon distribution functions (UGDF). The contribution of $\gamma^{*} \gamma^{*} \rightarrow \eta^{\prime}$ fusion is estimated. The distributions in the Feynman $x_{F}$ (or rapidity), transferred four-momenta squared between initial and final protons $\left(t_{1}, t_{2}\right)$ and azimuthal angle difference between outgoing protons $(\Phi)$ are calculated and discussed. The results are compared with the WA102 data. Predictions at higher energies are presented.

International Workshop on Diffraction in High-Energy Physics -DIFFRACTION 2006 September 5-10 2006

Adamantas, Milos island, Greece

\footnotetext{
* Speaker.

${ }^{\dagger}$ I am indebted to R. Pasechnik and O. Teryaev for collaboration on the subject of this presentation.
} 


\section{Introduction}

Recently the exclusive production of $\eta^{\prime}$ meson in proton-proton collisions was intensively studied close to its production threshold at the COSY ring at KFA Jülich [1] and at Saclay [2]. Here the dominant production mechanism is exchange of several mesons (so-called meson exchange currents) and reaction via $S_{11}$ resonance [3].

I present results of a recent study [4] (done in collaboration with R. Pasechnik and O. Teryaev) of the same exclusive channel but at much larger energies $(W>10 \mathrm{GeV})$. Here diffractive mechanism may be expected to be the dominant process. In Ref.[5] the Regge-inspired pomeron-pomeron fusion was considered as the dominant mechanism of the $\eta^{\prime}$ production. Here I present results obtained in the formalism based on unintegrated gluon distribution functions. Similar formalism was used recently to calculate cross section for exclusive Higgs boson production [6,7]. There is a chance that the formalism used for Higgs can be tested quantitatively for exclusive (heavy) meson production where the corresponding cross section is expected to be much bigger.

In Fig. 1 I show a sketch of the QCD mechanism of diffractive double-elastic production of $\eta^{\prime}$ meson (left diagram). For completeness, we include also photon-photon fusion mechanism (right diagram).
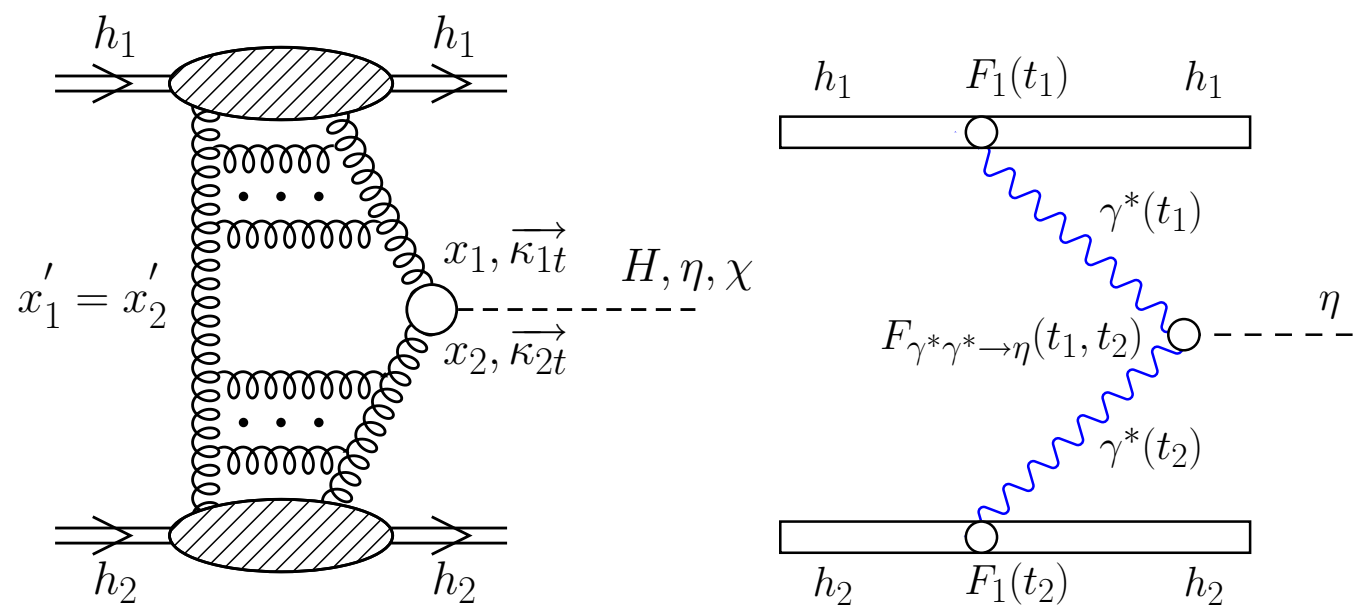

Figure 1: The sketch of the bare QCD and photon-photon fusion mechanisms. The kinematical variables are shown in addition.

\section{Formalism}

Following the formalism for the diffractive double-elastic production of the Higgs boson developed by Kaidalov, Khoze, Martin and Ryskin [6, 7] (KKMR) we write the bare QCD amplitude for the process $p p \rightarrow p \eta^{\prime} p$ sketched in Fig. 1 as

$$
\mathscr{M}_{p p \rightarrow p \eta^{\prime} p}^{g^{*} g^{*} \rightarrow \eta^{\prime}}=i \pi^{2} \int d^{2} k_{0, t} V\left(k_{1}, k_{2}, P_{M}\right) \frac{f_{g, 1}^{o f f}\left(x_{1}, x_{1}^{\prime}, k_{0, t}^{2}, k_{1, t}^{2}, t_{1}\right) f_{g, 2}^{o f f}\left(x_{2}, x_{2}^{\prime}, k_{0, t}^{2}, k_{2, t}^{2}, t_{2}\right)}{k_{0, t}^{2} k_{1, t}^{2} k_{2, t}^{2}} .
$$

The bare amplitude above is subjected to absorption corrections which depend on collision energy. The vertex function $V\left(k_{1}, k_{2}, P_{M}\right)$ in the expression (2.1) describes the coupling of two virtual glu- 
ons to the pseudoscalar meson. The details concerning the function $V\left(k_{1}, k_{2}, P_{M}\right)$ can be found in [4].

The objects $f_{g, 1}^{o f f}\left(x_{1}, x_{1}^{\prime}, k_{0, t}^{2}, k_{1, t}^{2}, t_{1}\right)$ and $f_{g, 2}^{o f f}\left(x_{2}, x_{2}^{\prime}, k_{0, t}^{2}, k_{2, t}^{2}, t_{2}\right)$ appearing in formula (2.1) are skewed (or off-diagonal) unintegrated gluon distributions. They are non-diagonal both in $x$ and $k_{t}^{2}$ space. Usual off-diagonal gluon distributions are non-diagonal only in $x$. In the limit $x_{1,2} \rightarrow x_{1,2}^{\prime}$, $k_{0, t}^{2} \rightarrow k_{1 / 2, t}^{2}$ and $t_{1,2} \rightarrow 0$ they become usual UGDFs. In the general case we do not know offdiagonal UGDFs very well. It seems reasonable, at least in the first approximation, to take

$$
\begin{aligned}
& f_{g, 1}^{o f f}\left(x_{1}, x_{1}^{\prime}, k_{0, t}^{2}, k_{1, t}^{2}, t_{1}\right)=\sqrt{f_{g}^{(1)}\left(x_{1}^{\prime}, k_{0, t}^{2}\right) \cdot f_{g}^{(1)}\left(x_{1}, k_{1, t}^{2}\right)} \cdot F_{1}\left(t_{1}\right), \\
& f_{g, 2}^{o f f}\left(x_{2}, x_{2}^{\prime}, k_{0, t}^{2}, k_{2, t}^{2}, t_{2}\right)=\sqrt{f_{g}^{(2)}\left(x_{2}^{\prime}, k_{0, t}^{2}\right) \cdot f_{g}^{(2)}\left(x_{2}, k_{2, t}^{2}\right)} \cdot F_{1}\left(t_{2}\right),
\end{aligned}
$$

where $F_{1}\left(t_{1}\right)$ and $F_{1}\left(t_{2}\right)$ are usual Dirac isoscalar nucleon form factors and $t_{1}$ and $t_{2}$ are total fourmomentum transfers in the first and second proton line, respectively. The above prescription is a bit arbitrary. It provides, however, an interpolation between different $x$ and $k_{t}$ values.

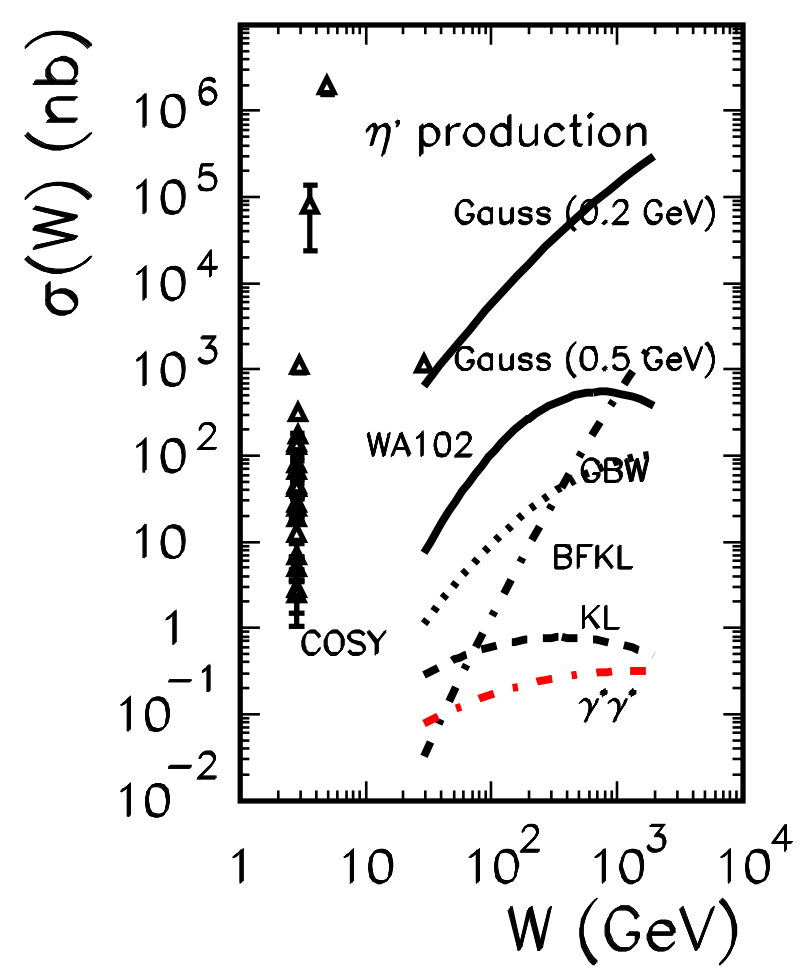

Figure 2: $\sigma_{t o t}$ as a function of center of mass energy for different UGDFs. The $\gamma^{*} \gamma^{*}$ fusion contribution is shown by the dash-dotted (red) line. The world experimental data are shown for reference.

Neglecting spin-flipping contributions the average matrix element squared for the $p\left(\gamma^{*}\right) p\left(\gamma^{*}\right) \rightarrow$ $p p \eta^{\prime}$ process can be written as [4]

$$
\overline{\left|\mathscr{M}_{p p \rightarrow p \eta^{\prime} p}^{\gamma^{*} \gamma^{*} \rightarrow \eta^{\prime}}\right|^{2}} \approx 4 s^{2} e^{8} \frac{F_{1}^{2}\left(t_{1}\right)}{t_{1}^{2}} \frac{F_{1}^{2}\left(t_{2}\right)}{t_{2}^{2}}\left|F_{\gamma^{*} \gamma^{*} \rightarrow \eta^{\prime}}\left(k_{1}^{2}, k_{2}^{2}\right)\right|^{2}\left|\mathbf{k}_{1, t}\right|^{2}\left|\mathbf{k}_{2, t}\right|^{2} \sin ^{2}(\Phi)
$$




\section{Results}

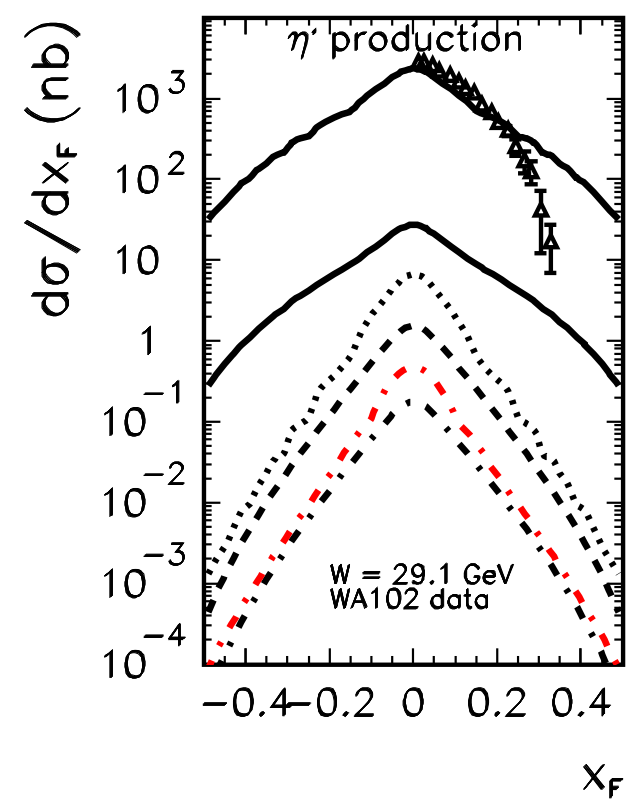

Figure 3: $d \sigma / d x_{F}$ as a function of Feynman $x_{F}$ for $\mathrm{W}=29.1 \mathrm{GeV}$ and for different UGDFs. The $\gamma^{*} \gamma^{*}$ fusion contribution is shown by the dash-dotted (red) line (second from the bottom). The experimental data of the WA102 collaboration [9] are shown for comparison.

In Fig. 2 I show energy dependence of the total (integrated over kinematical variables) cross section for the exclusive reaction $p p \rightarrow p \eta^{\prime} p$ for different UGDFs [8]. Quite different results are obtained for different UGDFs. This demonstrates huge sensitivity to the choice of UGDF. The cross section with the Kharzeev-Levin type distribution (based on the idea of gluon saturation) gives the cross section which is small and almost idependent of beam energy. In contrast, the BFKL distribution leads to strong energy dependence. The sensitivity to the transverse momenta of initial gluons can be seen by comparison of the two solid lines calculated with the Gaussian UGDF with different smearing parameter $\sigma_{0}=0.2$ and $0.5 \mathrm{GeV}$. The contribution of the $\gamma^{*} \gamma^{*}$ fusion mechanism (red dash-dotted line) is fairly small and only slowly energy dependent. While the QED contribution can be reliably calculated, the QCD contribution cannot be at present fully controlled.

In Fig. 3 I show the distribution of $\eta^{\prime}$ mesons in Feynman- $x_{F}$ obtained with several models of UGDF (for details see [8]). For comparison we show also the contribution of the $\gamma^{*} \gamma^{*}$ fusion mechanism. The contribution of the last mechanism is much smaller than the contribution of the diffractive QCD mechanism.

In Fig. 4 I present distribution in $t_{1}$ and $t_{2}$ (identical) of the diffractive production and of the $\gamma^{*} \gamma^{*}$ mechanism (red dash-dotted curve). The distribution for the $\gamma^{*} \gamma^{*}$ fusion is much steeper than that for the diffractive production. 


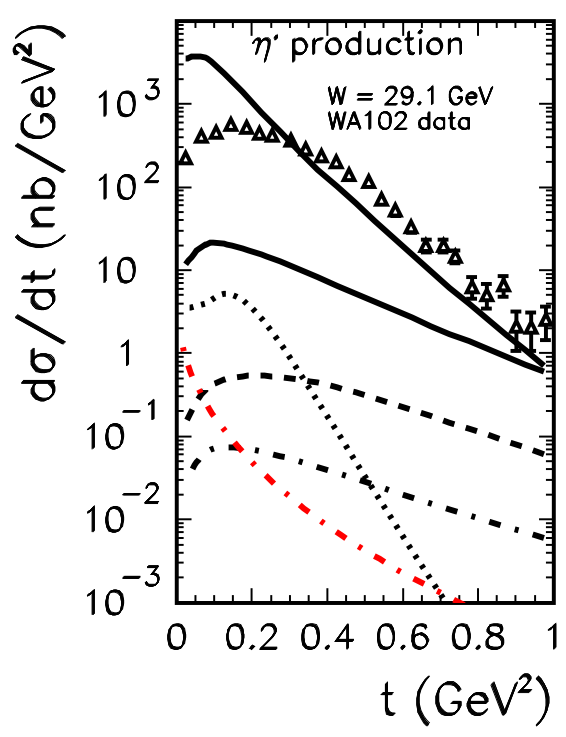

Figure 4: $d \sigma / d t_{1 / 2}$ as a function of Feynman $t_{1 / 2}$ for $\mathrm{W}=29.1 \mathrm{GeV}$ and for different UGDFs. The $\gamma^{*} \gamma^{*}$ fusion contribution is shown by the dash-dotted (red) steeply falling down line. The experimental data of the WA102 collaboration [9] are shown for comparison.

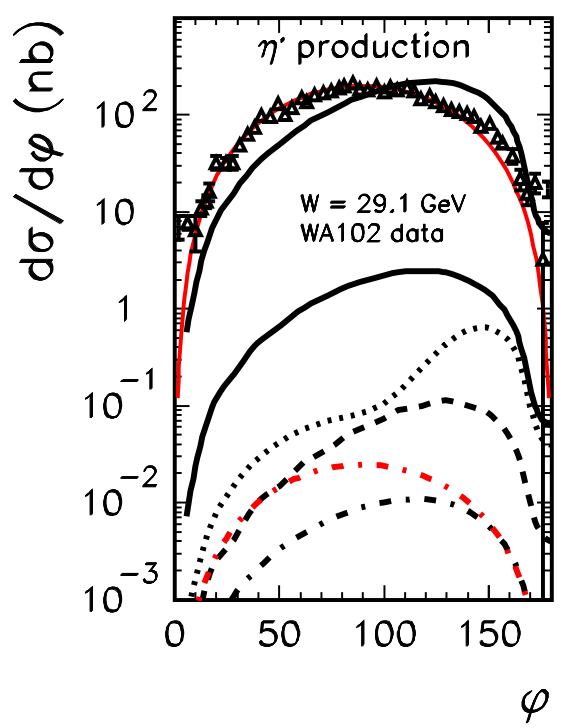

Figure 5: $\quad d \sigma / d \Phi$ as a function of $\Phi$ for $\mathrm{W}=29.1 \mathrm{GeV}$ and for different UGDFs. The $\gamma^{*} \gamma^{*}$ fusion contribution is shown by the dash-dotted (red) symmetric around $90^{\circ}$ line. The experimental data of the WA102 collaboration [9] are shown for comparison.

In Fig. 5 I show the distribution of the cross section as a function of the angle between the 
Table 1: Comparison of the cross section (in nb) for $\eta^{\prime}$ and $\eta_{c}$ production at Tevatron $(\mathrm{W}=1960 \mathrm{GeV})$ for different UGDFs. The integration is over $-4<y<4$ and $-1 \mathrm{GeV}<t_{1,2}<0$. No absorption corrections were included.

\begin{tabular}{|c|c|c|}
\hline UGDF & $\eta^{\prime}$ & $\eta_{c}$ \\
\hline KL & $0.4858(+0)$ & $0.7392(+0)$ \\
GBW & $0.1034(+3)$ & $0.2039(+3)$ \\
BFKL & $0.2188(+4)$ & $0.1618(+4)$ \\
Gauss $(0.2)$ & $0.2964(+6)$ & $0.3519(+8)$ \\
Gauss (0.5) & $0.3793(+3)$ & $0.4417(+6)$ \\
\hline$\gamma^{*} \gamma^{*}$ & $0.3095(+0)$ & $0.4493(+0)$ \\
\hline
\end{tabular}

outgoing protons. In the first approximation it reminds $\sin ^{2}(\Phi)$. A more detailed inspection shows, however, that the distribution is somewhat skewed with respect to $\sin ^{2}(\Phi)$ dependence.
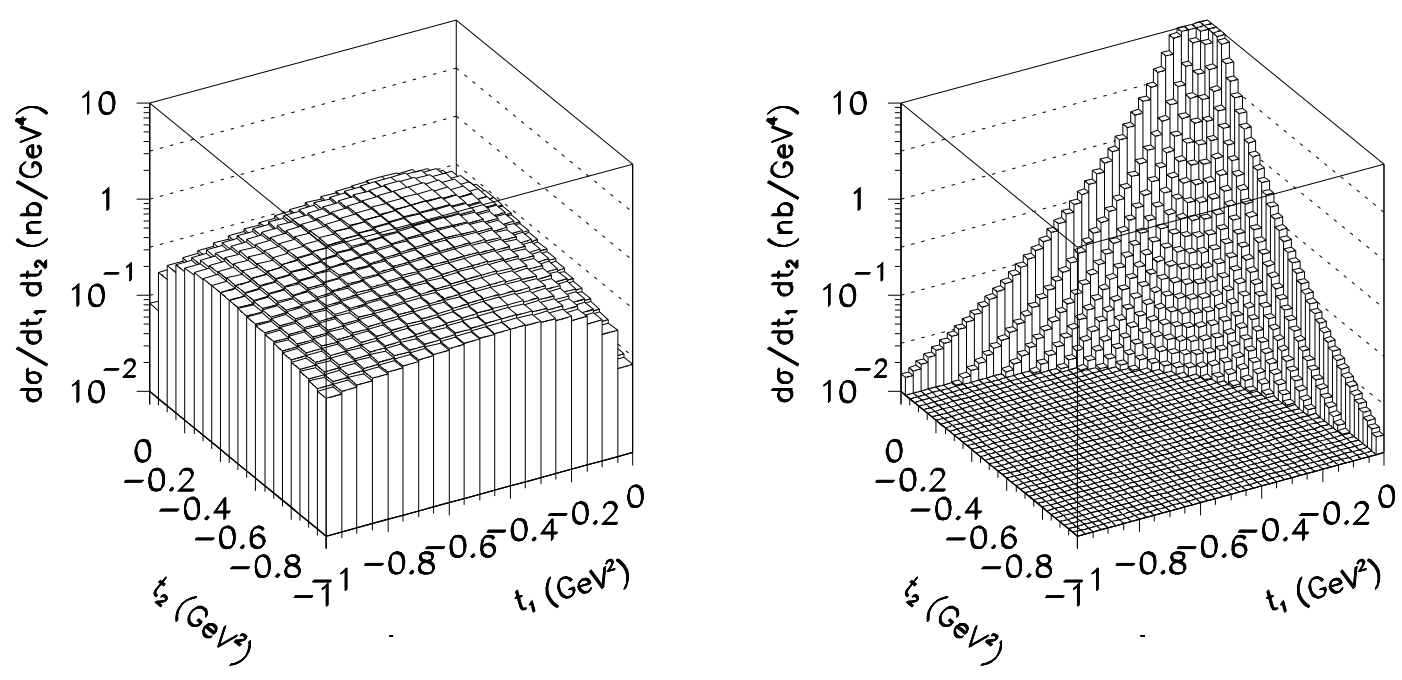

Figure 6: Two-dimensional distribution in $t_{1} \times t_{2}$ for the diffractive QCD mechanism (left panel), calculated with the KL UGDF, and the $\gamma^{*} \gamma^{*}$ fusion (right panel) at the Tevatron energy $\mathrm{W}=1960 \mathrm{GeV}$.

In Fig. 6 I present two-dimensional maps $t_{1} \times t_{2}$ of the cross section for the QCD mechanism (KL UGDF) and the QED mechanism (Dirac terms only) for the Tevatron energy W $=1960 \mathrm{GeV}$. If $\left|t_{1}\right|,\left|t_{2}\right|>0.5 \mathrm{GeV}^{2}$ the QED mechanism is clearly negligible. However, at $\left|t_{1}\right|,\left|t_{2}\right|<0.2 \mathrm{GeV}^{2}$ the QED mechanism may become equally important or even dominant. In addition, it may interfere with the QCD mechanism.

In Table 1 I have collected cross sections (in nb) for $\eta^{\prime}$ and $\eta_{c}$ mesons for $\mathrm{W}=1960 \mathrm{GeV}$ integrated over broad range of kinematical variables specified in the table caption. The cross sections for $\eta_{c}$ are very similar to corresponding cross sections for $\eta^{\prime}$ production and in some cases even bigger. 


\section{Conclusions}

I have shown that it is very difficult to describe the only exsisting high-energy ( $\mathrm{W} \sim 30 \mathrm{GeV}$ ) data measured by the WA102 collaboration [9] in terms of the unintegrated gluon distributions. First of all, rather large cross section has been measured experimentally. Using prescription (2.3) and on-diagonal UGDFs from the literature we get much smaller cross sections. Secondly, the calculated dependence on the azimuthal angle between the outgoing protons is highly distorted from the $\sin ^{2} \Phi$ distribution, whereas the measured one is almost a perfect $\sin ^{2} \Phi$ [4]. This signals that a rather different mechanism plays the dominant role at this energy. Exchange of subleading reggeons is a plausible mechanism.

The diffractive QCD mechanism and the photon-photon fusion lead to quite different pattern in the $\left(t_{1}, t_{2}\right)$ space. Measuring such two-dimensional distributions at Tevatron and/or LHC would certainly help in disentangling the reaction mechanism.

Finally we have presented results for exclusive double elastic $\eta_{c}$ production. Similar cross sections as for $\eta^{\prime}$ production were obtained. Also in this case the results depend strongly on the choice of UGDF.

Measurements of the exclusive production of $\eta^{\prime}$ and $\eta_{c}$ would help to limit or even pin down the UGDFs in the nonperturbative region of small gluon transverse momenta where these objects cannot be obtained as a solution of any perturbative evolution equation, but must be rather modelled.

\section{References}

[1] P. Moskal et al. (COSY11 collaboration), Phys. Rev. Lett. 80 (1998) 3202; P. Moskal et al. (COSY11 collaboration), Phys. Lett. B474 (2000) 416. P. Moskal et al. (COSY11 collaboration), Phys. Lett. B482 (2000) 356.

[2] F. Balestra et al. (DISTO collaboration), Phys. Lett. B491 (2000) 29.

[3] K. Nakayama and H. Haberzettl, Phys. Rev. C69 (2004) 065212.

[4] A. Szczurek, R.S. Pasechnik and O.V. Teryaev, hep-ph/0608302.

[5] N.I. Kochelev, T. Morii and A.V. Vinnikov, Phys. Lett. B457 (1999) 202.

[6] V.A. Khoze, A.D. Martin and M.G. Ryskin, Phys. Lett. B401 (1997) 330; V.A. Khoze, A.D. Martin and M.G. Ryskin, Eur. Phys. J. C23 (2002) 311; A.B. Kaidalov, V.A. Khoze, A.D. Martin and M.G. Ryskin, Eur. Phys. J. C31 (2003) 387; A.B. Kaidalov, V.A. Khoze, A.D. Martin and M.G. Ryskin, Eur. Phys. J. C33 (2004) 261.

[7] J. Forshaw, hep-ph/0508274.

[8] M. Łuszczak and A. Szczurek, Phys. Rev. D73 (2006) 054028.

[9] D. Barberis et al. (WA102 collaboration), Phys. Lett. B422 (1998) 399. 